\title{
A choice-semantical approach to theoretical truth
}

\author{
Holger Andreas \\ Georg Schiemer
}

Penultimate Draft

\begin{abstract}
A central topic in the logic of science concerns the proper semantic analysis of theoretical sentences, that is sentences containing theoretical terms. In this paper, we present a novel choice-semantical account of theoretical truth based on the epsilonterm definition of theoretical terms. Specifically, we develop two ways of specifying the truth conditions of theoretical statements in a choice functional semantics, each giving rise to a corresponding logic of such statements. In order to investigate the inferential strength of these logical systems, we provide a translation of each truth definition into a modal definition of theoretical truth. Based on this, we show that the stronger notion of choice-semantical truth captures more adequately our informal semantic understanding of scientific statements.
\end{abstract}

\section{Introduction}

A central topic in the logic of science concerns the proper semantic analysis of theoretical terms and theoretical sentences. How, if at all, do theoretical terms refer to their objects? In what ways do the theoretical postulates of a theory contribute to the specification of the meaning of these terms? Finally, how should we evaluate semantically scientific statements that contain theoretical terms? Carnap and Ramsey were the first to address these question using formal tools, thus providing the foundation for virtually all subsequent research on the semantics of theoretical terms (cf. [22], [32], [27], [16], [31], etc.).

Let us explain the challenges of a semantic analysis of theoretical terms with a simple example, taken from collision mechanics. Suppose we have two spherical objects $s_{1}$ and 
$s_{2} . s_{2}$ is at rest at time $t$, whereas $s_{1}$ moves toward $s_{2}$ with a certain velocity $v_{1}$. Then, $s_{1}$ collides with $s_{2}$ to the effect that $s_{1}$ is at rest at time $t^{\prime}\left(t^{\prime}>t\right)$, whereas $s_{2}$ moves with the velocity $v_{2}^{\prime}=v_{1}$ at $t^{\prime}$. In other words, the first spherical objects transfers its momentum to the second by an elastic collision. As is well known, this experiment lets us infer that the two objects must have the same mass.

How can we draw the distinction between theoretical and observational concepts in this experiment? Arguably, the concept of mass is theoretical because we understand this concept in terms of scientific theories, such as collision mechanics and classical mechanics. The metrical concepts of space, time and velocity may be argued to be theoretical as well because our understanding of these concepts depends on certain measurement theories. However, we can take these concepts as (relatively) observational in the context of classical and collision mechanics because classical and collision mechanics are not needed to understand space, time, and velocity in non-relativistic physics.

The challenge arising here is to devise a semantics that explains how classical and collision mechanics shape the meaning of the concept of mass. Ideally, this semantics should specify rules for the assignment of truth-values to statements about the mass of the two objects in our experiment. What may be described as standard semantics fails to account for the genuine semantic properties of theoretical terms. For when using a logical language $\mathcal{L}$ for the representation of statements about some domain, it is standard to assume a complete and direct extensional interpretation of the descriptive vocabulary of $\mathcal{L}$. Such an interpretation fails to display the semantic dependency of theoretical terms on a scientific theory.

Logical accounts of theoretical terms have in common that the scientific theory in question somehow constrains the interpretation of these terms. This constraint leaves some degree of indeterminacy as it does not result in a unique interpretation of theoretical terms. In our simple collision experiment, the interpretation of theoretical terms is constrained by the laws of elastic collisions, i.e. conservation of momentum as well as conservation of energy. Given the values of the velocities of the objects, before and after the collision, these two conservation laws are verified by a range of interpretations of the concept of mass. Hence, we have an indeterminacy of interpretation of the concept of mass. Yet, we want to say that certain statements about the mass of the two bodies are true, while others are false. By conservation of momentum, we know that $m_{1}=m_{2}$ must 
come out true, whereas $m_{1}<m_{2}$ should come out false; $m_{1}$ and $m_{2}$ designate the mass of the two objects, respectively.

The question thus arises how the truth-values of the statements about the mass of the two objects are determined by a range of interpretations. This is the key question to be addressed in what follows. We shall explain in detail how a scientific theory determines a range of interpretations of the theoretical terms and how these interpretations, in turn, determine the semantic values of theoretical statements.

\section{Two logical accounts of theoretical terms}

In Carnap's logic of science we can recognize two logical accounts of theoretical terms. One centers around the notion of an indirect and partial interpretation of theoretical terms $([6,7,8])$. Roughly, this account states that the interpretation of the theoretical terminology of a scientific theory is not specified directly through metatheoretic definitions but indirectly through the postulates of the theory. ${ }^{1}$ The second account originates from the Ramsey sentence and makes use of Hilbert's epsilon operator, thereby aiming at an explicit definition of theoretical terms ([9, 29]). ${ }^{2}$ Whereas the former account is couched in semantic terms, the latter is purely syntactic. Neither approach, however, gives us an explication of theoretical truth, i.e. a fully-fledged semantics of theoretical statements.

The present paper has two principal objectives. The first one is to provide an explicit model-theoretic analysis of the epsilon account of theoretical terms based on a choicefunctional semantics for the epsilon operator ([21, 4, 24]). In particular, we shall develop two different choice-semantical approaches to the semantics of theoretical statements, each giving rise to a corresponding logic of such statements. The second objective of the paper is to investigate the inferential strength of the two logics underlying the different choice-semantical approaches to theoretical truth. This will be done by relating them to recent work on the model-theoretic explication of Carnap's indirect interpretation view of

\footnotetext{
${ }^{1}$ See [3] for a survey of different model-theoretic explications of this view in the modern literature.

${ }^{2}$ Compare [30] for a systematic study of the epsilon reconstruction of theories and its compatibility with scientific structuralism.
} 
theoretical terms. ${ }^{3}$ More precisely, we shall exploit the modal reconstruction of theoretical truth by Andreas [2]. According to this account, a scientific sentence (containing both observational and theoretical terms) is theoretically true if and only if it is true in all admissible extensions of its intended observational model, that is, in all model extensions that interpret the theoretical terms in accordance with the theory's axioms. This modal explication turns out to be equivalent with the particular choice-semantical explication of theoretical truth to be developed here. ${ }^{4}$

Moreover, the equivalence between the modal explication of the indirect interpretation view of theoretical terms and the choice functional account of the epsilon reconstruction of theoretical knowledge also shows that the two accounts are conceptually similar in several respects. In particular, the meaning of theoretical terms is specified contextually, i.e. relative to a given theoretical context in both approaches. Moreover, both accounts make precise in different ways a central intuition driving systematic work on theoretical terms since Carnap, viz., that the determination of such terms by a scientific theory remains incomplete (see [2, 30]).

The paper will be organized as follows: Section 3 briefly outlines the epsilon-term reconstruction of scientific theories as well as the epsilon logic and choice semantics underlying it. Based on this, we present two possible model-theoretic explications of theoretical truth in line with Carnap's general approach. Section 4 will then turn to a closer comparison between these choice-semantical explications and the above mentioned modal account of theoretical truth. By means of this comparison, we shall investigate the inferential strength of the two choice-semantical explications of theoretical truth in Section 5 . Finally, Section 7 will give a summary of our findings.

\section{Choice semantics and theoretical truth}

In this section, we develop a choice-semantical account of theoretical truth based on Carnap's epsilon-term definition of theoretical terms [9, 29]. According to the syntactic (or received) view of theories, a scientific theory $T$ can be expressed in a higher-order

\footnotetext{
${ }^{3}$ Compare [16] for a detailed discussion of this account of theoretical terms and its historical development in Carnap's work.

${ }^{4}$ The modal explication is inspired by supervaluation logic, as readers will easily recognize in Section 4
} 
language $\mathcal{L}\left(V_{o}, V_{t}\right)$ that contains a set of observational terms $V_{o}$ and a set of theoretical terms $V_{t}$. To keep the discussion simple, we will assume that the descriptive vocabulary consists only of unary predicates and relational symbols. A complex sentence TC of this language is usually said to express the conjunction of the axioms of $T$ (see, e.g., [2, 20]).

Carnap's logical reconstruction of theories is based on two steps. ${ }^{5}$ The first one is the elimination of the theoretical terms in $\mathcal{L}\left(V_{0}, V_{t}\right)$ by the ramsification of a theory. As is well know, the Ramsey sentence of a given $T$ is constructed by substituting its theoretical terms by existentially quantified variables of the proper logical category:

$$
\exists X_{1} \ldots \exists X_{n} T C\left(X_{1}, \ldots, X_{n}, O_{1}, \ldots O_{m}\right)
$$

The Ramsey sentence-henceforth abbreviated by $R S(T)$ - is expressed in the "observational" sublanguage $\mathcal{L}\left(V_{o}\right)$ of the theoretical language $\mathcal{L}\left(V_{o}, V_{t}\right)$. Roughly speaking, it says that there exist theoretical relations in the universe of the language that have the features which the theory attributes to them.

Carnap's second step consists in the subsequent reintroduction of the theoretical vocabulary through an explicit definition in language $\mathcal{L}\left(V_{o}\right)$ supplemented by a logical $\epsilon$-operator. The $\epsilon$-operator was originally introduced in Hilbert's proof-theoretic work in the 1920s. ${ }^{6}$ It functions syntactically as a term-forming operator: if $A(x)$ is a formula with $x$ occurring as free first-order variable in it, then $\epsilon_{x} A(x)$ is a term where all occurrences of $x$ are bound. This term picks out an arbitrary object from the extension of $A$ in case this extension is non-empty. Applied to formulas with free higher-order variables, the epsilon operator functions in a similar way. In the present context, we restrict our attention to predicate variables (of finite arities): let $A(X)$ be a formula with one free $n$-ary predicate variable $X$ occurring in it. The expression $\epsilon_{X} A(X)$ then presents a higher-order term that picks out an arbitrary $n$-ary relation on the domain of the model in which statement $A$ is true.

This operator is applied in [9] in the following way: let $\bar{T}=\left\langle T_{1}, \ldots, T_{n}\right\rangle$ stand for the $n$-ary tuple of all theoretical terms described by $T$. Then the explicit definition of this tuple has the following form:

\footnotetext{
${ }^{5}$ See also [30] for a more detailed presentation of the epsilon reconstruction of scientific theories.

${ }^{6}$ See [5] and [35] for discussions of the historical development of the epsilon logic. Compare [21], [24] and [36] for presentations of the epsilon calculus.
} 


$$
\bar{T}:=\epsilon_{Z} \exists X_{1} \ldots \exists X_{n}\left(Z=\left\langle X_{1}, \ldots, X_{n}\right\rangle \wedge T C\left(X_{1}, \ldots, X_{n}, O_{1}, \ldots O_{m}\right)\right)
$$

Informally speaking, the theoretical symbols are defined here as referring to an arbitrary tuple of relations that, if assigned to the free variables in $R S(T)$, make the Ramsey sentence true. Put differently, the definition picks out one arbitrary sequence of extensions of the theoretical vocabulary in case the theory in question is internally consistent and consistent with the empirical facts.

Based on this general definition of the tuple of all theoretical terms, the extension of each singular term $T_{i}$ (for $1 \leq i \leq n$ ) can then be specified relative to this particular choice (if $T_{i}$ has an extension), namely as the $i$-th element of tuple $\bar{P}_{t}$ :

$$
T_{i}:=\epsilon_{Y} \exists X_{1} \ldots \exists X_{n}\left(\bar{T}=\left\langle X_{1}, \ldots, X_{n}\right\rangle \wedge Y=X_{i}\right)^{7}
$$

The original presentation of the epsilon reconstruction of theories in [9] is purely syntactic in character. Nonetheless, Carnap's account motivates a semantic analysis of theoretical terms that captures closely his informal remarks about the indefinite or indeterminate nature of such terms. Notice that, in his account, theoretical terms do not have a direct interpretation but are defined indirectly in terms of the observational terminology of a theory. How is their interpretation specified according to Carnap's proposal? Specifically, how does the epsilon-term definition fix a reference of these terms?

To see this, we have to look more closely at the semantics underlying the use of epsilonterms here. The standard semantic treatment of epsilon languages is based on a choicefunctional interpretation of the epsilon operator. ${ }^{8}$ Such a choice semantics for an extensional $\epsilon$ logic can be characterized in the following way: ${ }^{9}$ an interpretation $\mathcal{M}$ of a language $\mathcal{L}_{\epsilon}$ has the form $\langle D, I\rangle$, where $D$ is a domain and $I$ an interpretation function for the nonlogical vocabulary. The $\epsilon$-symbol can be interpreted relative to a given choice

\footnotetext{
${ }^{7}$ Notice that the 'local' definitions of individual theoretical terms are relativized to the prior definition of tuple $\bar{P}_{t}$. See $[9$ for details.

${ }^{8}$ Carnap's work does not contain an explicit model-theoretic specification of the $\epsilon$-language used by him. It is clear, however, that he was familiar with a choice functional interpretation of epsilon terms. There is a remark in [9] that the epsilon-symbol presents a selection function. Moreover, Carnap refers to [4] for a specification of the epsilon calculus and its choice semantics, i.e. "a semantical (i.e. set-theoretic) interpretation in terms of logical selection functions (a function of this kind assigns to each non-empty subset of the domain of individuals an elements of this subset)" [9. p.156].

${ }^{9}$ We follow closely here Zach's presentation of a choice semantics for an extensional epsilon calculus presented in [36]. For a more detailed study of the semantics of epsilon terms (including a semantics for intensional epsilon logic based on a universe of Skolem functions) see [24].
} 
function based on this model. ${ }^{10}$ Specifically, depending on the logical category of the variable from which an epsilon expression is constructed, epsilon symbols can refer to functions of different types. For the first order case, a choice function is a function of the form $\delta: \wp(D) \rightarrow D$ such that, for any $X \subseteq D$ :

$$
\delta(X)= \begin{cases}x \in X, & \text { if } X \neq \varnothing \\ x \in D & \text { otherwise. }\end{cases}
$$

A choice function thus picks out a "representative" object for any non-empty set and an arbitrary object from domain $D$ if the set in question is empty.

In the case of second-order epsilon terms built from $n$-ary predicate variables, a suitable choice function will be of the form $\Delta: \wp\left(\wp\left(D^{n}\right)\right) \rightarrow \wp\left(D^{n}\right)$ such that, for any set of relations $\operatorname{Rel} \subseteq \wp\left(D^{n}\right)$, we have:

$$
\Delta(\text { Rel })= \begin{cases}X \in R e l, & \text { if } \operatorname{Rel} \neq \varnothing ; \\ X \in \wp\left(D^{n}\right) & \text { otherwise. }\end{cases}
$$

Given this account of extensional choice functions, closed $\epsilon$-terms are interpreted relative to a structure $\mathcal{M}$, an assignment function $s$ to first-order and predicate variables, and choice functions $\delta, \Delta$ on $\mathcal{M}$ based on the following two valuation rules: ${ }^{11}$

$$
\begin{aligned}
\text { val }^{\mathcal{M}, \delta, s}\left(\epsilon_{x} A(x)\right) & =\delta\left(\text { val }^{\mathcal{M}, s}(A(x))\right) \\
& =\delta(\{d \in D \mid \mathcal{M}, s[x / d] \models A(x)\}) \\
\text { val }^{\mathcal{M}, \Delta, s}\left(\epsilon_{X} A(X)\right)= & \Delta\left(\text { val }^{\mathcal{M}, s}(A(X))\right) \\
=\Delta & \left(\left\{R \in \wp\left(D^{n}\right) \mid \mathcal{M}, s[X / R] \models A(X)\right\}\right)
\end{aligned}
$$

These rules state that the referent of an $\epsilon$-term constructed from $A$ is the element (or relation) that the choice function picks from the set of objects (or relations) defined by formula $A$ in model $\mathcal{M}$.

The choice-functional treatment of epsilon logic gives us a way to make precise the

\footnotetext{
${ }^{10}$ The epsilon-symbol is treated as a functional variable here that effectively ranges over possible choice functions on a given model. This variable choice conception is first developed in Asser: "Das Zeichen $\epsilon$ schlielich ist eine Variable für Auswahlfunktionen des Individuenbereichs J" [4, p.33]. See [21] for a different account.

${ }^{11}$ We restrict our attention here to valuation rules for the special case of closed epsilon terms. For a more general treatment of valuation rules for epsilon terms with parameters, see [24, pp.33-34].
} 
informal semantics underlying the present account of theoretical terms and theoretical statements. In order to do so, we have to describe the epsilon reconstruction of theories also from a model-theoretic perspective. Let us assume in the following that language $\mathcal{L}\left(V_{o}, V_{t}\right)$ is interpreted relative to a single domain of individuals. All variables of the language range over domain $D$ or over higher-order domains constructible from it. ${ }^{12}$ An important assumption concerning the descriptive vocabulary is that the observational predicates in set $V_{o}$ have a fixed and intended interpretation. Thus, we assume that the statements of sublanguage $\mathcal{L}\left(V_{o}\right)$ are fully interpreted in an "observational" or base model $\mathcal{O}=\left\langle D, \bar{R}_{o}\right\rangle$. This model contain a number of observational relations $\bar{R}_{o}$ on domain $D$ that function as the intended interpretations of the observational predicates in $\mathcal{L}\left(V_{o}\right)$.

By contrast, the theoretical vocabulary of the language is usually considered to be uninterpreted at first. More specifically, its interpretation is specified only indirectly by the axioms of the theory expressed in $\mathcal{L}\left(V_{o}, V_{t}\right)$. This fact can be elucidated by looking at how the Ramsey sentence of a theory is evaluated semantically. What are the truth conditions of the Ramsey sentence of a theory? Informally speaking, we say that $R S(T)$ is true if and only if there exists at least one set of theoretical relations on the domain that make the theory true. More formally, $R S(T)$ is true in the base model $\mathcal{O}$, i.e. $\left\langle D, \bar{R}_{o}\right\rangle \models R S(T)$, if and only if there exists at least one ordered sequence of theoretical relations $\overline{R_{t}}$ on $D$ such that the expanded structure $\mathcal{B}=\left\langle D, \bar{R}_{o}, \bar{R}_{t}\right\rangle$ satisfies the axioms of the theory, i.e. $\left\langle D, \bar{R}_{o}, \bar{R}_{t}\right\rangle \mid=T C$, where $I\left(T_{i}\right)^{\mathcal{B}}=R_{i}$, for each $i \in n .{ }^{13}$

An interpretation of the theoretical terms is thus specified relative to an expansion of the base model by relations that satisfy the theoretical axioms in question. We call an $\mathcal{L}\left(V_{o}, V_{t}\right)$-structure admissible if it is an expansion of the observational model $\mathcal{O}$ that satisfies the axioms of $T$ (cf. [2], [3]).

Assuming that the Ramsey sentence of a theory is satisfied, it follows that the epsilonterm definition picks out an arbitrary instance of such a tuple of relations for the interpretation of the theoretical terms. Notice that the definiens in the epsilon-term definition scheme contains the "variabilized" theory, that is an open formula of the form

\footnotetext{
${ }^{12}$ We will also assume that the higher-order quantifiers of $\mathcal{L}\left(V_{0}, V_{t}\right)$ have a standard interpretation. In particular, predicate variables are supposed to range over the full powerset $\wp\left(D^{n}\right)$, for each arity $n$.

${ }^{13}$ See [20, p.293] and [14].
} 


$$
\mathrm{TC}\left(\mathrm{X}_{1}, \ldots, \mathrm{X}_{n}, \mathrm{O}_{1}, \ldots \mathrm{O}_{m}\right)
$$

This formula defines the class of interpretations of the theoretical predicates of language $\mathcal{L}\left(V_{o}, V_{t}\right)$ relative to theory $T$ :

$$
\operatorname{Ext}\left(V_{t}\right)^{T}:=\left\{\bar{R}_{t}\left|\left\langle D, \bar{R}_{o}, \bar{R}_{t}\right\rangle\right|=T C\right\}
$$

This is, informally speaking, the class of all extensional interpretations of the theoretical vocabulary that, if joined with the given observational model, result in full models of $T .^{14}$

An epsilon term in the above definition can be evaluated semantically in the base model $\mathcal{O}$ and relative to a particular choice function constructible from it. Such a function assigns a particular member of the class $\operatorname{Ext}\left(V_{t}\right)^{T}$ to that class. ${ }^{15}$ The interpretation of theoretical terms can then be specified relative to such a higher-order choice function. Specifically, we can specify the interpretation of the tuple $\bar{T}$ of predicates in $\mathcal{L}\left(V_{o}, V_{t}\right)$ based on a semantic valuation of its defining epsilon term relative to model $\mathcal{O}$, assignment function $s$ and higher-order choice function $\Delta$ in the following way:

$$
\begin{aligned}
I(\bar{T}) & =v_{a l}^{\mathcal{O}, \Delta, s}\left(\epsilon_{\bar{X}} \operatorname{TC}(\bar{X}, \bar{O})\right) \\
& =\Delta\left(v a l^{\mathcal{O}, s}(T C(\bar{X}, \bar{O}))\right) \\
& =\Delta\left(\left\{\overline{R_{t}} \mid \mathcal{O}, s\left[\bar{X} / \overline{R_{t}}\right]=T C(\bar{X}, \bar{O})\right\}\right)
\end{aligned}
$$

The reference of the theoretical predicates in a full model of the language is specified here in terms of the choice-functional interpretation of their defining $\epsilon$-term, namely as an arbitrary sequence of relations that make the ramsified theory true in model $\mathcal{O}$.

The choice semantics for $\epsilon$-languages not only gives us a precise account of the reference of theoretical terms that nicely corresponds to Carnap's purely syntactic approach. It also provides the means for a model-theoretic analysis of the semantics of theoretical sentences, where a sentence qualifies as theoretical if and only if it contains theoretical

\footnotetext{
${ }^{14}$ Alternatively, we can take $\operatorname{Ext}\left(V_{t}\right)^{T}$ to be the class of possible assignments to the 'theoretical' variables of the observational language that make RS(T) true in $\mathcal{O}$.

${ }^{15}$ To give a simple example, let $V_{t}$ contain only one binary predicate $P(x, y)$. The class $\operatorname{Ext}\left(V_{t}\right)^{T}$ (for a given theory $T$ ) will then consist of relations of type $R \subseteq D \times D$. A suitable choice function will then be a function of the form: $\Delta: \wp\left(\wp\left(D^{2}\right)\right) \rightarrow \wp\left(D^{2}\right)$ such that$$
\Delta\left(\operatorname{Ext}\left(V_{t}\right)^{T}\right)= \begin{cases}X \in \operatorname{Ext}\left(V_{t}\right)^{T}, & \text { if } \operatorname{Ext}\left(V_{t}\right)^{T} \neq \varnothing ; \\ X \in \wp\left(\wp\left(D^{2}\right)\right) & \text { otherwise. }\end{cases}
$$ 
terms. As is shown in [21, 4, 24, 36], one can define a general notion of truth for statements of $\mathcal{L}_{\epsilon}$ based on the standard valuation and satisfaction rules for predicate logic and the valuation rules for epsilon terms stated above. We will express the fact that the formula $A$ (containing epsilon terms) is satisfied in model $\mathcal{M}$, relative to a choice function $\delta$ and an assignment function $s$ by $\mathcal{M}, \delta, s \mid=A$.

A semantic evaluation of theoretical statements based on this choice-functional notion of satisfaction comes in two steps: In the first step, a translation of language $\mathcal{L}\left(V_{o}, V_{t}\right)$ in the observational language supplemented by an epsilon operator $\mathcal{L}_{\epsilon}\left(V_{o}\right)$ has to be specified that maps all theoretical sentences to their $\epsilon$-term translations, i.e. to sentences gained by the systematic substitution of $t$-terms by their defining $\epsilon$-terms. Let $\varphi$ be a sentence in a theoretical language that contains a number of theoretical predicates (and possibly also observational predicates). Then, given the suggested definition scheme for theoretical terms, there is a function that maps the theoretical sentence $\varphi$ to a sentence $\varphi^{\epsilon}$ in which each occurrence of a term $T_{i}$ in $\varphi$ is substituted by its defining $\epsilon$-term. More formally, a relative translation of language $\mathcal{L}\left(V_{o}, V_{t}\right)$ in $\mathcal{L}_{\epsilon}\left(V_{o}\right)$ can be given by a mapping $F$ that associates each theoretical relation symbol $T_{i}$ (of arity $m$ ) with its epsilon-operator definition in the above specified sense. ${ }^{16}$ Based on this, a translation function $\varphi^{\epsilon}$ for $\mathcal{L}\left(V_{o}, V_{t}\right)$ sentences $\varphi$ can be specified inductively in the following way:

$$
\begin{aligned}
& \left(T_{i}\left(t_{1}, \ldots, t_{m}\right)\right)^{\epsilon}:=\left\langle t_{1}, \ldots, t_{m}\right\rangle \in \epsilon_{Y} \exists X_{1} \ldots \exists X_{n}\left(\bar{T}=\left\langle X_{1}, \ldots, X_{n}\right\rangle \wedge Y=X_{i}\right) \\
& (\varphi \wedge \psi)^{\epsilon}:=\varphi^{\epsilon} \wedge \psi^{\epsilon} ;(\varphi \vee \psi)^{\epsilon}:=\varphi^{\epsilon} \vee \psi^{\epsilon} ;(\neg \varphi)^{\epsilon}:=\neg \varphi^{\epsilon} \\
& (\exists x \varphi)^{\epsilon}:=\exists x(\varphi)^{\epsilon} ;(\forall x \varphi)^{\epsilon}:=\forall x(\varphi)^{\epsilon}
\end{aligned}
$$

Given this relative translation, the second step is then to evaluate the resulting $\epsilon$-translation $\varphi^{\epsilon}$ of each theoretical statement in terms of the choice-functional semantics presented above. As should be clear, $\varphi^{\epsilon}$ can be interpreted in the base model $\mathcal{O}$ and relative to a suitable choice function on it.

Given this, we can give an explication of the truth of theoretical sentences in choicefunctional terms. Specifically, we can distinguish between two types of theoretical truth based on two different truth conditions for epsilon-term statements given in [24]. Let $\varphi \in \mathcal{L}\left(V_{o}, V_{t}\right)$, let $\varphi^{\epsilon}$ be its $\epsilon$-translation in $\mathcal{L}_{\epsilon}\left(V_{0}\right)$ based on theory $T$.

\footnotetext{
${ }^{16}$ Observational predicates remain invariant under this translation. Moreover, the translation is unrelativized in the sense that the range of the quantifiers remains identical.
} 


\section{Definition 1 (Local truth and generic truth of $\varphi^{\epsilon}$ ) We say that}

1. $\varphi^{\epsilon}$ is locally true if and only if there exists a choice function $\delta$ with respect to $\mathcal{O}$ such that $\mathcal{O}, \delta \mid=\varphi^{\epsilon}$;

2. $\varphi^{\epsilon}$ is generically true (or valid) if and only if for every choice function $\delta$ with respect to $\mathcal{O}$, we have $\mathcal{O}, \delta=\varphi^{\epsilon}{ }^{17}$

Falsehood is understood in terms of the truth of the negated sentence: $\varphi^{\epsilon}$ is locally false if and only if $\neg \varphi^{\epsilon}$ is locally true. $\varphi^{\epsilon}$ is generically false if and only if $\neg \varphi^{\epsilon}$ is generically true. $^{18}$

Why do we distinguish between these two notions of truth for epsilon-translations of theoretical sentences? The semantic values of all epsilon translations of theoretical sentences are indeterminate if no relativization to some choice function or a range of choice functions is assumed. This cannot be an intended result for the semantics of theoretical sentences because we do assert and deny theoretical sentences in science. The content of such assertions and denials should be represented by a proper semantics of theoretical sentences. Hence, epsilon translations of theoretical sentences should be understood relative to choice functions.

There seem to be at least three "natural" options for relativizing the semantics of epsilontranslations to choice functions. First, truth relative to a particular choice function $\delta$ with respect to $\mathcal{O}$. Second, local truth as just defined. Third, generic truth as just defined. The first option might be in the spirit of the epsilon operator, but it would make the semantics of theoretical sentences dependent upon an arbitrary choice of a particular choice function. No such dependence is implied by the latter two options. Hence, it is reasonable to pursue these options.

The challenge arising here is to decide which, if any, of the two notions of truth explicates the content of our assertions of theoretical sentences in science. In the next two

\footnotetext{
${ }^{17}$ The terms local and generic truth are introduced in Meyer Viol's study of intensional and extensional epsilon logic in [24]. A similar distinction can already be found in [4]. Notice, moreover, that the local truth of theoretical statements so construed is closely related to the notion of partial truth discussed in the framework of partial structures by [12]. It is easy to show that the local truth of $\varphi^{\epsilon}$ is definable in terms of the partial truth of $\varphi$ in a simple pragmatic structure.

${ }^{18}$ Notice that a given formula $\varphi^{\epsilon}$ can be both locally true (i.e. true relative to a given choice function) and locally false (relative to another choice function). This problematic fact will be discussed in further detail in Section 5
} 
sections, we shall answer this question by comparing the present approach with another, well established model-theoretic explication of the semantics of theoretical terms. This is Andreas' modal (or supervaluationist) account of theoretical truth ([1], [2]). Based on his approach, we will propose a modal translation of local truth and generic truth, respectively. This translation enables us to recognize two different logics to which the two different notions of truth give rise. It turns out that only one of the two logics is inferentially strong enough to account for scientific reasoning.

\section{A modal translation}

As was shown in the previous section, the explicit epsilon-term definition

$$
\bar{T}={ }_{d f} \epsilon_{Z} \exists X_{1} \ldots \exists X_{n}\left(Z=\left\langle X_{1}, \ldots, X_{n}\right\rangle \wedge T C\left(X_{1}, \ldots, X_{n}, O_{1}, \ldots O_{m}\right)\right)
$$

leaves us with a range of admissible interpretations of the theoretical terms:

$$
\operatorname{Ext}\left(V_{t}\right)^{T}:=\left\{\bar{R}_{t} \mid\left\langle D, \bar{R}_{o}, \bar{R}_{t}\right\rangle=\mathrm{TC}\right\}
$$

Together with the fixed interpretation $\bar{R}_{o}$ of the observational predicates, any member of $\operatorname{Ext}\left(V_{t}\right)^{T}$ defines a complete interpretation of $\mathcal{L}\left(V_{o}, V_{t}\right)$. We can conceive of these interpretations as possible worlds:

$$
W:=\left\{\left\langle D, \bar{R}_{o}, \bar{R}_{t}\right\rangle\left|\left\langle D, \bar{R}_{o}, \bar{R}_{t}\right\rangle\right| T C\right\}
$$

Figuratively speaking, any world $w \in W$ represents a way things may be like, assuming the interpretation $\bar{R}_{o}$ of the observational predicates and the truth of the Ramsey sentence $R S(T)$. Let us now adopt a standard S5 modal semantics, where we have $R=W \times W$ for the accessibility relation $R$. As we do not distinguish between interpretations of $\mathcal{L}\left(V_{o}, V_{t}\right)$ and worlds, our first order modal model of $\mathcal{L}\left(V_{o}, V_{t}\right)$ has the following simple structure:

$$
\mathfrak{M}=\langle W, R\rangle
$$

Arguably, this modal model formally represents the interpretation of theoretical terms in line with [8]. For, first, the postulates TC indirectly interpret the theoretical terms by constraining their interpretation: TC must be true in any possible world. This constraint does not yield a complete interpretation of the theoretical terms, however. Their interpretation remains incomplete in the sense of leaving a certain degree of indeterminacy: 
any of the possible worlds $w \in W$ represents an admissible interpretation.

We are now in a position to define two alternative notions of theoretical truth: ${ }^{19}$

Definition 2 (Semantics of theoretical sentences) Let $\varphi$ be an $\mathcal{L}\left(V_{o}, V_{t}\right)$ sentence :

1. $\varphi$ is weakly theoretically true if and only if $\mathfrak{M}=\diamond \varphi$.

2. $\varphi$ is strongly theoretically true if and only if $\mathfrak{M}=\square \varphi$.

That is, an $\mathcal{L}\left(V_{o}, V_{t}\right)$ sentences $\varphi$ is weakly theoretically true if and only if it is true in at least one world $w$ of $W$. It is strongly theoretically true if and only if it is true in all worlds $w$ of $W$. In other words, strong theoretical truth means that the sentence is true in all interpretations being admitted by the postulates and by the given interpretation of $\mathcal{L}\left(V_{o}\right)$. These truth-rules are inspired by supervaluation logic and we are deeply indebted to Priest's exposition of this logic in [28]. The notion of strong theoretical truth formally coincides with supertruth, while weak theoretical truth coincides with subtruth (cf. Williamson's account of vagueness [34, pp. 142-153]).

For $\mathcal{L}\left(V_{o}\right)$ sentences $\varphi$, the two notions of truth coincide, provided the Ramsey sentence $R S(T)$ is true. Furthermore, on condition of $R S(T)$, the conjunction $T C$ of postulates is both strongly and weakly theoretically true. However, there clearly are $\mathcal{L}\left(V_{o}, V_{t}\right)$ sentences $\varphi$ for which the two notions of theoretical truth diverge from one another. This follows from $W$ not being a singleton. Recall that $W$ cannot be singleton because this would contradict the doctrine that the interpretation of theoretical terms is incomplete. ${ }^{20}$

The two modal notions of theoretical truth translate, respectively, the semantics of theoretical sentences that we have defined using choice functions in the preceding section:

Observation 1 Let $\varphi$ be a sentence of $\mathcal{L}\left(V_{0}, V_{t}\right)$.

1. $\varphi$ is locally true if and only if $\mathfrak{M}=\diamond \varphi$.

2. $\varphi$ is generically true if and only if $\mathfrak{M}=\square \varphi$.

\footnotetext{
${ }^{19}$ Strong theoretical truth has been suggested as an explication of theoretical truth in [2].

${ }^{20}$ It follows from the incompleteness of the determination of theoretical terms that, for any theoretical term $T_{i}$, there are at least two admissible interpretations of $\mathcal{L}\left(V_{o}, V_{t}\right)$ that interpret $T_{i}$ differently. Let us call them $w_{1}$ and $w_{2}$. Hence, there is a theoretical sentence $\varphi$ such that $w_{1}=\varphi$ and $w_{2} \not \models \varphi$. Therefore, if $W$ is the set of admissible interpretations represented by the modal model $\mathfrak{M}$, we have $\mathfrak{M} \models \diamond \varphi$ but $\mathcal{M} \not \models \square \varphi$.
} 
This observation follows easily from the definition of $\mathfrak{M}$ and the way in which choicefunctional truth was specified in Section 3 . Recall from above that a theoretical statement was said to be locally true if there exists at least one choice function on the observational model that picks out an admissible interpretation of the theoretical vocabulary in question. In turn, a theoretical statement was said to be generically true if and only if every possible choice function on the observational base model picks out an admissible interpretation of these terms.

As we saw, one way to characterize whether an interpretation is theoretically admissible, i.e. admissible relative to a given axiomatic theory, can be given in terms of the notion of model expansions. We said that an interpretation of theoretical predicates is admissible if the expanded structure $\mathcal{B}$ resulting from model $\mathcal{O}$ by the addition of these theoretical relations satisfies the theory in question. Given this, the two types of choice-theoretical truth can be characterized in the following way: Let $\varphi^{\epsilon}$ be an $L_{\epsilon}\left(V_{o}\right)$ sentence. Then $\varphi^{\epsilon}$ is weakly theoretically true if and only if there exists (at least) one choice function $\delta$ on $\mathcal{O}$ such that $I(\bar{T})=\bar{R}_{t}=v_{a l}{ }^{\mathcal{O}, \delta}\left(\epsilon_{\bar{X}} T C(\bar{X}, \bar{O})\right)$ and $\left\langle D, \bar{R}_{o}, \bar{R}_{t}\right\rangle=\varphi(\bar{T}, \bar{O})$. Put differently, we say that $\varphi^{\epsilon}$ is partially true if and only if one can construct an admissible expansion $\mathcal{B}$ from $\mathcal{O}$ based on a choice function $\delta$ such that $\mathcal{B}=\varphi$. Since model $\mathcal{B}$ is by definition a possible world in $W$, it follows that local truth is equivalent to weak theoretical truth (in the modal sense).

Similarly, we say that the epsilon-translate $\varphi^{\varepsilon}$ of a theoretical statements is generically true if and only if for every possible choice function $\delta$ on $\mathcal{O}$ such that $I\left(\bar{P}_{t}\right)=\bar{R}_{t}=$ val $^{\mathcal{O}, \delta}\left(\epsilon_{\bar{X}} T(\bar{X}, \bar{O})\right)$ we have $\left\langle D, \bar{R}_{o}, \bar{R}_{t}\right\rangle \models \varphi\left(\bar{P}_{t}, \bar{O}\right)$. Thus, the sentence $\varphi^{\epsilon}$ is generically true if and only if $\varphi$ is true in all possible expansions of $\mathcal{O}$ to full models of theory $T$ that are constructed in the above sense. This is again equivalent to our definition of strong theoretical truth as truth in all possible worlds of $\mathfrak{M}$.

\section{Inferential strength}

In this section, we shall show that strong theoretical truth yields a more reasonable semantics of theoretical sentences than its weak alternative. This result carries over directly to the alternative between local and generic truth within the choice semantical treatment. Our two modal notions of theoretical truth give rise to two different logics insofar as they 
define two different relations of logical consequence:

Definition $3\left(A=_{w}\left(\mid=_{s}\right) \varphi\right)$ Let $A$ be a set of $\mathcal{L}\left(V_{0}, V_{t}\right)$ sentences and $\varphi$ be such a sentence. Let $={ }_{S 5}$ be the $S 5$ consequence relation. $\square A(\diamond A)$ is the set obtained from $A$ by prefixing any member of $A$ with $\square(\diamond)$.

1. $A=_{w} \varphi$ if and only if $\diamond A \mid={ }_{S 5} \diamond \varphi$.

2. $A=_{S} \varphi$ if and only if $\square A={ }_{S 5} \square \varphi$.

It turns out that $\left.\right|_{s}$-the consequence relation of strong theoretical truth-is equivalent to the relation of logical consequence in classical logic ([2]):

Proposition 1 Let $A$ and $\varphi$ be as above. $F_{c l}$ is the relation of logical consequence in classical logic. Then, $A=_{s} \varphi$ if and only if $A={ }_{c l} \varphi$.

Proof. Suppose $A \models_{s} \varphi$. Then, by definition of $\models_{s}, \square A \models_{S 5} \square \varphi$. That is, for all S5 models $\mathfrak{M}$, if $\mathfrak{M} \models \square \alpha$ for all $\alpha \in A$, then $\mathfrak{M}=\square \varphi$. Therefrom it follows that, for all S5 models $\mathfrak{M}$ for which $W$ is a singleton, if $\mathfrak{M}=\square \alpha$ for all $\alpha \in A$, then $\mathfrak{M}=\square \varphi$. Hence, for all classical models $\mathcal{M}$, if $\mathcal{M} \models A$, then $\mathcal{M}=\varphi$. Thus, $A \models{ }_{c l} \varphi$.

For the other direction, suppose (i) $A=_{c l} \varphi$. Further, let us assume that (ii), for a set $\mathbf{M}$ of classical models, if $\mathcal{M} \in \mathbf{M}$, then $\mathcal{M} \models A$. (i) and (ii) imply that (iii), for all $\mathcal{M} \in \mathbf{M}$, $\mathcal{M}=\varphi$. From (ii) and (iii) we can infer that for all sets $\mathbf{M}$ of classical models $\mathcal{M}$ : if, for all $\mathcal{M} \in \mathbf{M}, \mathcal{M} \models A$, then, for all $\mathcal{M} \in \mathbf{M}, \mathcal{M} \models \varphi$. Hence, for all S5 models $\mathfrak{M}$, if $\mathfrak{M}=\square \alpha$ for all $\alpha \in A$, then $\mathfrak{M} \models \square \varphi$. By definition of $=_{{ }_{s}}$, it follows therefrom that $A \mid={ }_{s} \varphi$.

Proposition 1 is clearly a desirable result in the analysis of informal deductive reasoning in science if one wants to retain (as Carnap did) constant theoretical terms as opposed to higher order variables. The Ramsey sentence itself, though semantically illuminating, provides a very cumbersome formulation of a theory that is unsuitable as analysis of scientific reasoning (cf.[16]). It may be argued, moreover, that virtually all inference rules of classical natural deduction are needed to account for scientific reasoning. ${ }^{21}$ Hence, it is a desirable result that the present logic of theoretical truth retains these inference rules. Further extensions of this logic, such as logics of nonmonotonic and abductive reasoning, may be built on top of this classical core.

\footnotetext{
${ }^{21}$ Disjunction introduction remains a controversial case, though.
} 
The consequence relation of weak theoretical truth, however, is much less well behaved. For example, it is easy to show that $\models_{w}$ fails to validate modus ponens. If modus ponens were valid for $=_{w}$, the following inference rule would have to be valid in S5:

$$
\frac{\diamond \alpha \quad \diamond(\alpha \rightarrow \beta)}{\diamond \beta}
$$

A counterexample to this inference rule is easy to construct: let $L(V)$ be a language of propositional modal logic with $V=\{p, q\}$. Let, further $W$ (the set of worlds) be given by $\left\{w_{1}, w_{2}\right\}$. Suppose, in $w_{1} p$ and $\neg q$ hold true, whereas in $w_{2} \neg p$ and $\neg q$ are true. Then, it holds that $\diamond p, \diamond(p \rightarrow q)$ but not $\diamond q$. Hence, there is a propositional modal $S 5$ model for which modus ponens fails to be valid. This propositional model can easily be transformed into a first order modal model of the modal extension of $\mathcal{L}\left(V_{0}, V_{t}\right)$. Simply assume that $q$ is an $\mathcal{L}\left(V_{0}, V_{t}\right)$ sentence that is false in all $w \in W$. $p$, by contrast, may be true in some $w \in W$ and false in some other world $w^{\prime} \in W$.

In a similar vein, it can be shown that other important inference rules of natural deduction with two premises fail to hold for weak theoretical truth. This is particularly unfavorable for an account of scientific reasoning in the case of the following inference rule of first order logic with identity, which is sometimes referred to as the Leibniz principle:

$$
\frac{s=t \quad \varphi[s]}{\varphi[t / s])} \quad \operatorname{free}(s, t, \varphi[*])
$$

This inference rule says that, if $t=s$ and $\varphi, t$ can be substituted for $s$ in $\varphi$ at all occurrences of $s$ where $s$ is free and $t$ does not become bounded if substituted for $s$. The Leibniz principle is used whenever we insert one equation into another; it is thus of utmost importance in physics and other sciences using mathematical equations. This principle is not valid in the logic of weak theoretical truth, however. It fails to be valid because $s=t$ and $\varphi$ may, respectively, be true at different worlds, while there is no world at which both $s=t$ and $\varphi$ are true.

In sum, the consequence relation of weak theoretical truth fails to validate inferential patterns that are at the core of scientific reasoning. We must therefore realize that weak theoretical truth does not explicate well the semantics of theoretical sentences. The semantics of strong theoretical truth, by contrast, lets us preserve the validity of classical deductive reasoning. This is a desirable result, not only because of Carnap's adherence 
of classical logic. For, even though classical deductive reasoning may not capture all of the inference patterns used in science, classical logic continues to serve as the basis of a logical analysis of science. Various more refined accounts of scientific reasoning, such as nonmonotonic logics and systems of abductive reasoning, have been defined on this basis. Moreover, the patterns of classical deductive logic seem to be needed for an account of pure and applied mathematical reasoning. Hence, strong theoretical truth scores much better than weak theoretical truth in terms of inferential properties.

Even though the logic of strong theoretical truth is inferentially equivalent with classical logic, the notion of strong theoretical truth does not satisfy the principle of bivalence. That is, there are $\mathcal{L}\left(V_{0}, V_{t}\right)$ sentences $\varphi$ such that $\varphi$ is neither strongly theoretically true nor strongly theoretically false. More precisely, $\varphi$ is indeterminate if and only if $\mathfrak{M} \models \diamond \varphi$ and $\mathfrak{M}=\diamond \neg \varphi$. It is not obvious whether this is a problem at all. Arguably, the existence of indeterminate theoretical sentences is a consequence of the incompleteness of the interpretation of the theoretical terms.

In the case of weak theoretical truth, every $\mathcal{L}\left(V_{0}, V_{t}\right)$ sentence is true or false. However, some $\mathcal{L}\left(V_{0}, V_{t}\right)$ sentences are both true and false. More precisely, theoretical sentences $\varphi$ being indeterminate as regards strong theoretical truth are both weakly theoretically true and weakly theoretically false. Hence, we have truth-value gluts in the case of weak theoretical truth. This implies that weak theoretical truth does not satisfy the principle of bivalence either. It is very questionable, moreover, whether the truth-value gluts of weak theoretical truth capture any aspect of our understanding of theoretical truth. Hence, weak theoretical truth does not lead to a more plausible truth-value assignment than strong theoretical truth.

This not very surprising "victory" of strong theoretical truth over its weak alternative helps us decide between the two notions of truth that we have distinguished within the choice semantics of the epsilon operator. By Observation 1 and Proposition 1, we know that generic truth (as defined by Definition 1) is inferentially equivalent with classical logic. Observation 1 furthermore, lets us conclude that local truth (as defined by Definition 1) suffers from the problem of inferential weakness in the same way as weak theoretical truth does. That is, local truth fails to validate inferential patterns that are at the core of scientific reasoning, such as modus ponens and the Leibniz principle. It is therefore inferentially too weak to account for scientific reasoning properly. 
Furthermore, the notion of generic truth yields a more plausible assignment of truthvalue to theoretical sentences. The fact that some $\mathcal{L}\left(V_{0}, V_{t}\right)$ sentences remain indeterminatein the sense of being neither generically true nor generically false-reflects the incompleteness of the interpretation of theoretical terms. With local truth, by contrast, we have truth-value gluts even for scientific theories $T C$ that are classically consistent. This does not seem to be a desirable property.

In sum, there are two important criteria that lead us to favor the notion of generic truth within the choice semantics. First, generic truth is inferentially stronger than local truth to the extent that it is inferentially equivalent with classical logic. Second, generic truth comes with a more plausible truth-value assignment than local truth. Hence, we opt for the following choice semantics as an explication of theoretical truth:

Explication 1 (Theoretical truth) Let $\varphi$ be an $\mathcal{L}\left(V_{0}, V_{t}\right)$ sentence and $\varphi^{\epsilon}$ its epsilon translation. $\varphi$ is true if and only if for all choice functions $\delta$ with respect to $\mathcal{O}$ such that $\mathcal{O}, \delta=\varphi^{\epsilon}$.

\section{Inferential properties exemplified}

Let us return to our collision experiment to exemplify the inferential weakness of local truth. Recall that the interpretation of mass is merely constrained by the laws of conservation of energy and conservation of momentum as well as the velocities of the objects before and after the collision. Given these constraints, it is easy to show that for any real number $x$ there is a choice function such that $m_{1}=x$. Likewise for $m_{2}$. Hence, the following theoretical statements are locally true:

$$
m_{1}=5 \quad m_{2}=7
$$

Now take the following inference:

$$
\begin{array}{rrr}
m_{1}=5 \quad m_{2}=7 \quad 5<7 \\
m_{1}<m_{2}
\end{array}
$$

The conclusion can be inferred using the Leibniz principle twice. This inference, however, is not valid according to the semantics of local truth. For there is no choice function such that $m_{1}<m_{2}$ comes out locally true. Hence, the Leibniz principle is not valid for local 
truth.

A similar problem does not arise for generic truth because $m_{1}=5$ and $m_{2}=7$ are not generically true for obvious reasons. Moreover, the fact that $m_{1}=x$ is locally true for any arbitrary value $x$ speaks against explicating theoretical truth in terms of local truth. Such statements cannot be inferred from the measurements of our collision experiment. Hence, they should not be true on our account of theoretical truth. By contrast, $m_{1}=m_{2}$ is inferable from the measurements, which is generically true, as it should be.

\section{Conclusion}

We have described two different candidates for a choice-semantics of theoretical truth in this paper. A central philosophical motivation for such a semantics is to account for the indefinite nature of the interpretation of theoretical terms. Recall that explicit definition of such terms via epsilon terms merely constrains the interpretation of such terms but does not individuate their reference. A choice function can then be understood to pick out one determinate but arbitrary interpretation from the range of interpretations that satisfy the constraint by TC.

As we saw, this choice semantic framework leaves us with at least two different options for explicating the semantics of theoretical sentences. The first option has been described as local truth: A theoretical statement is true if and only if there exists at least one suitable choice function $\delta$ such that the epsilon translation of this statement is true relative to the observational model and to $\delta$. The second option has been described as generic truth: A theoretical statement is true if and only if its epsilon translation is true relative to the observational model and all possible choice functions (of the relevant type).

Given this framework, the question arises which of the two notions captures the semantics of theoretical sentences in a more appropriate way? We have answered this question by translating the two notions under consideration into the language of a simple S5 modal semantics and a subsequent investigation of the inferential properties of the translates.

It turns out that the logic of generic truth is inferentially equivalent to classical logic, which is a desirable result. Local truth, by contrast, is inferentially too weak to account 
for scientific reasoning. It could be shown, moreover, that the truth-value assignment of generic truth is more plausible than that of local truth for two reasons. First, the former, unlike the latter, avoids truth-value gluts. Second, the semantics of theoretical truth reflects the incompleteness of the interpretation of theoretical terms. Local truth, by contrast, may be described as overdeterminate because there are theoretical sentences being both locally true and locally false.

\section{References}

[1] H. Andreas. Another solution to the problem of theoretical terms. Erkenntnis, 69:315-333, 2008.

[2] H. Andreas. A modal view of the semantics of theoretical sentences. Synthese, 174:367-383, 2010.

[3] H. Andreas. Theoretical terms in science. The Stanford Encyclopedia of Philosophy, http://plato.stanford.edu/archives/sum2013/entries/theoreticalterms-science/, 2013.

[4] G. Asser. Theorie der logischen Auswahlfunktionen. Zeitschrift für mathematische Logik und Grundlagen der Mathematik, 3:30-68, 1957.

[5] J. Avigad and R. Zach. The Epsilon Calculus. In E.N. Zalta, editor, The Stanford Encyclopedia of Philosophy. http:// plato.stanford.edu/archives/win2013/entries/epsiloncalculus/, 2013.

[6] R. Carnap. Foundations of Logic and Mathematics. International encyclopedia of unified science, vol.I. Chicago: University of Chicago Press, 1939.

[7] R. Carnap. The methodological character of theoretical concepts. In H. Feigel and M. Scriven, editors, Minnesota studies in the philosophy of science, vol. I: The foundations of science and the concepts of psychology and psychoanalysis, volume 1, pages 38-76. Minneapolis: University of Minnesota, 1956.

[8] R. Carnap. Beobachtungssprache und theoretische Sprache. Dialectica, 12(236-248), 1958. 
[9] R. Carnap. On the use of Hilbert's $\epsilon$-operator in scientific theories. In Y. Bar-Hillel et al., editor, Essays on the foundations of mathematics, pages 156-164. Jerusalem: The Magnus Press, 1961.

[10] R. Carnap. Replies and systematic expositions. In P. A. Schilpp, editor, The philosophy of Rudolf Carnap, pages 859-1013. LaSalle: Open Court, 1963.

[11] N. da Costa, O. Bueno, and S. French. The logic of pragmatic truth. Journal of Philosophical Logic, 27(6):603-620, 1998.

[12] N. da Costa and S. French. Science and Partial Truth. Oxford: OUP, 2003.

[13] W. Demopoulos. On the rational reconstruction of our theoretical knowledge. British Journal for the Philosophy of Science, 54(371-403), 2003.

[14] W. Demopoulos. Logicism and its Philosophical Legacy. Cambridge: Cambridge University Press, 2013.

[15] M. Friedman. Wissenschaftslogik: The role of logic in the philosophy of science. Synthese, 164:385-400, 2008.

[16] M. Friedman. Carnap on theoretical terms: structuralism without metaphysics. Synthese, 180:249-263, 2011.

[17] C. G. Hempel. The theoretician's dilemma: A study in the logic of theory construction. In M. Scriven H. Feigl and G. Maxwell, editors, Minnesota studies in the philosophy of science, vol. II: Concepts, theories and the mind-body problem, pages 37-98. Minneapolis: University of Minnesota Press, 1958.

[18] C. G. Hempel. The meaning of theoretical terms: A critique of the standard empiricist construal. In A. Joja P. Suppe, L. Henkin and G. C. Moisil, editors, Logic, Methodology and Philosophy of Science IV, pages 367-378. Amsterdam, 1973.

[19] D. Hilbert and P. Bernays. Grundlagen der Mathematik. Springer, 1934/1939.

[20] J. Ketland. Empirical adequacy and ramsification. British Journal for the Philosophy of Science, 55(287-300), 2004.

[21] A. C. Leisenring. Mathematical Logic and Hilbert's Epsilon-Symbol. London: Macdonald, 1969. 
[22] D. Lewis. How to define theoretical terms. The Journal of Philosophy, 67(13):427-446, 1970.

[23] G. Maxwell. Structural realism and the meaning of theoretical terms. In S. Winokur and M. Radner, editors, Minnesota Studies in the Philosophy of Science, vol. IV: Analyses of Theories and Methods of Physics and Psychology, pages 181-192. Minneapolis: University of Minnesota Press, 1970.

[24] W. P. M. Meyer Viol. Instantial Logic. An Investigation into Reasoning with Instances. ILLC Dissertation Series 1995-11. ILLC, Amsterdam, 1995.

[25] G. M. Mints and D. Sarenac. Completeness of an indexed epsilon-calculus. Archive for Mathematical Logic, 42:617-625, 2003.

[26] G. Moser and R. Zach. The epsilon calculus and Herbrand complexity. Studia Logica, 82(1):133-155, 2006.

[27] D. Papineau. Theory-dependent terms. Philosophy of Science, 63:1-20, 1996.

[28] G. Priest. An Introduction to Non-Classical Logic. Cambridge University Press, Cambridge, 2001.

[29] S. Psillos. Rudolf Carnap's 'Theoretical concepts in science'. Studies in History and Philosophy of Science, 31:151-172, 2000.

[30] G. Schiemer and N. Gratzl. The epsilon reconstruction of theories and scientific structuralism. Erkenntnis, DOI 10.1007/s10670-015-9747-9.

[31] Gerhard Schurz. Criteria of theoreticity: Bridging statement and non-statement view. Erkenntnis, 79(8):1521-1545, 2014.

[32] J. Sneed. The Logical Structure of Mathematical Physics. D. Reidel Publishing Company, Dordrecht, 1979.

[33] B. van Fraassen. Presuppositions, supervaluations and free logic. In K. Lambert, editor, The Logical Way of Doing Things, pages 67-92. Yale University Press, New Haven, 1969.

[34] T. Williamson. Vagueness. Routledge, London, 1994. 
[35] R. Zach. The practice of finitism. Epsilon calculus and consistency proofs in Hilbert's Program. Synthese, 137:211-259, 2003.

[36] R. Zach. Lectures on the epsilon calculus. Lecture notes, http://arxiv.org/abs/1411.3629, 2009. 